\title{
Determination of the number of unpaired electrons in metal-complexes. A comparison between the Evans'Method and susceptometer results.
}

\author{
${ }^{1}$ K. De Buysser ${ }^{*},{ }^{1}$ G.G.Herman, ${ }^{1}$ E. Bruneel, ${ }^{1}$ S. Hoste and ${ }^{1}$ I. Van Driessche \\ ${ }^{1}$ Department of Inorganic and Physical Chemistry, Ghent University, \\ Krijgslaan 281, Building S3, 9000 Ghent, Belgium \\ E-mail : Klaartje.DeBuysser@Ugent.be
}

\begin{abstract}
. 1,4,8,11-tetraazacyclotetradecane (cyclam) is widely known as an ideal ligand for chelating heavy metal ions such as $\mathrm{Ni}^{2+}$ and $\mathrm{Cu}^{2+}$ in aqueous media. In this work the consequences of chelation on the preference for high spin or low spin configuration were investigated for $\mathrm{Fe}^{3+}, \mathrm{Ni}^{2+}, \mathrm{Cu}^{2+}$ and $\mathrm{Cr}^{3+}$. Two methods were used to determine the number of unpaired electrons in the solid and dissolved complex. First the change of magnetic susceptibility of the crystalline powder as a function of temperature was measured. In the second case Evans NMR-based method was used to obtain information about the number of unpaired electrons in the dissolved complexes. In some cases, such as $\mathrm{Fe}^{3+}$ and $\mathrm{Ni}^{2+}$, a discrepancy between the two methods was noticed. This behaviour is discussed in terms of the preference of the large high spin $\mathrm{Ni}^{2+}$-ion to switch to the smaller low spin $\mathrm{Ni}^{2+}$-ion in the aqueous environment, which better fits the cavity of the ligand in the square planar geometry. The results are influenced by the geometry of the complex, the aggregation state and the dimensions of the ions.
\end{abstract}

Keywords: NMR method, 1,4,8,11-tetraazacyclotetradecane, high spin, low spin

\section{Introduction}

The interest for the synthesis of a large number of metallated heterocycles started in the early sixties. They were the foundation of many research fields such as biological studies or metalion catalysis, leading towards industrial and medical applications.[1] Cyclic polyamines have long been known to form stable complexes with various guests.[2] In the last decades many studies have focussed on the surface modification of several supports for use in waste water treatment.[3] Our interest in the influence of chelating on the electron configuration of the metal salts is based on an exploration of magnetic separation techniques using superconducting magnets and leads us to the measurement of their magnetic properties. Hence, we applied NMR in a formalism known as the Evans'method to the measurement of magnetic susceptibility directly in aqueous media.[4]

\section{Subject and Methods}

\section{Chemicals}

$\mathrm{CuCl}_{2} .2 \mathrm{H}_{2} \mathrm{O}, \mathrm{CrCl}_{3} .6 \mathrm{H}_{2} \mathrm{O}, \mathrm{FeCl}_{2} \cdot 4 \mathrm{H}_{2} \mathrm{O}$ were purchased from UCB Chemicals (Belgium). $\mathrm{FeCl}_{3} \cdot 6 \mathrm{H}_{2} \mathrm{O}$, t-butyl alcohol, $\mathrm{D}_{2} \mathrm{O}$, dimethylsulfoxide, methanol and chloroform were obtained from Chem-Lab nv (Belgium). $\mathrm{NiCl}_{2} \cdot 6 \mathrm{H}_{2} \mathrm{O}$ was bought from JT Baker Chemicals (Holland). Cyclam was obtained from Aldrich (Germany). Liquid nitrogen, Dioxygen gas en Argon gas were purchased from Air Liquide Company. Chemicals were used as received without further purifications except for chloroform and t-butyl alcohol. Chloroform was dried over activated 
molecular sieves $(4 \AA)$, t- butyl alcohol was purified by destillation at $82^{\circ} \mathrm{C}$. The metal-cyclam complexes were synthesized as previously reported.[5-7]

\section{Instrumentation}

The NMR-analysis was conducted on a Varian, Gemini $300 \mathrm{MHz}$, at room temperature , 300 $\mathrm{MHz}, 32$ scans. The susceptibility measurements were performed using a Lakeshore 7000 series susceptometer. The frequency was $125 \mathrm{~Hz}$ and the applied field was $2000 \mathrm{~A} / \mathrm{m}$. The temperature was increased from $80 \mathrm{~K}$ to $120 \mathrm{~K}$, rate $0,5 \mathrm{~K} \mathrm{sec}{ }^{-1}$. UV-Vis spectra were recorded on a Varian Cary 50 spectrophotometer.

\section{Calculation of the number of unpaired electrons in paramagnetic complexes}

The equations used for the calculation of the number of unpaired electrons are mentioned below. In the case of the solid complex, susceptometer measurements were done as a function of temperature. Following the Curie law and using the spin-only approximation of the magnetic moment leads us towards Eq. 1. This equation makes it possible to do a simple calculation of the unpaired electrons in the solid complexes.

$$
\mu_{e f f}=\sqrt{\frac{3 k}{N \mu_{b}^{2} \mu_{0}}} \sqrt{\chi_{M} T}=798 \sqrt{\chi_{M} T}
$$

The Evans technique is applicable to solutions and is based on the frequency shift occasioned in an NMR signal of a co-dissolved compound, t-butyl alcohol, by the additional magnetic field due to the accompanying paramagnetic species. A coaxial double NMR tube is used.

The relative frequency shift $\Delta v / v$ with $\Delta v=v_{\text {alcohol }}-\left(v_{\text {alcohol }}\right.$ complex $)$ produced by the presence of a metal species is used to caculate the magnetic moment by Eq. 2 for a superconducting solenoid magnet.

$$
\mu_{e f f}=798 \sqrt{\chi_{M} T}=798 \sqrt{\left(-\frac{3 \Delta v}{v m}+\chi_{\text {mass }, 0}\right) \cdot M W}
$$

\section{Results}

Using Eq. 2, the magnetic moment for all the complexes was determined according the NMR method. After dissolving the metal salts or complexes in the $\mathrm{D}_{2} \mathrm{O}$ - $\mathrm{t}$-butyl alcohol mixtures, the spectra are recorded and the relative shift of the reference peaks is calculated. The number of unpaired electrons can readily be calculated and the results are listed in table 1 . The theoretically expected number of unpaired electrons, is also mentioned in this table.[8]

Table 1. Results obtained by Evans method and analysis of the solid complex.

\begin{tabular}{cccc}
\hline Name of compound & Unpaired e (theor.) & $\begin{array}{l}\text { Liquid } \\
\text { complex }\end{array}$ & $\begin{array}{l}\text { Solid } \\
\text { complex }\end{array}$ \\
\hline $\mathrm{CrCl}_{3} \cdot 6 \mathrm{H}_{2} \mathrm{O}$ & 3 & 3,02 & 2,69 \\
$\mathrm{Cr}(\mathrm{III}) \mathrm{cycl}$ & 3 & 2,81 & 3,62 \\
$\mathrm{FeCl}_{3} \cdot 6 \mathrm{H}_{2} \mathrm{O}$ & 5 & 4,49 & 7,37 \\
cis-Fe(III)cycl & 5 & 4,87 & 7,68 \\
trans-Fe(II)cycl & 1 & 3,56 & 2,71 \\
$\mathrm{NiCl}_{2} \cdot 6 \mathrm{H}_{2} \mathrm{O}$ & 2 & 2,32 & 2,06 \\
$\mathrm{Ni}(\mathrm{II}) \mathrm{cycl}_{\mathrm{CuCl}} \cdot 2 \mathrm{H}_{2} \mathrm{O}$ & 2 & 1,06 & 1,77 \\
$\mathrm{Cu}(\mathrm{II}) \mathrm{cycl}$ & 1 & 1,20 & 1,01 \\
& 1 & 0,93 & 0,61 \\
\hline
\end{tabular}


The results agree remarkably well except for the trans-Fe-cyclam complex. This complex should exhibit low spin behaviour due to the square planar surrounding. The two chloride ions are in the axial position, leading to $\mathrm{D}_{4 \mathrm{~h}}$ symmetry as confirmed by X-ray analysis.[7] The result of 3.38 unpaired electrons indicates that the $\mathrm{FeCl}_{4}{ }^{-}$ion which makes part of the complex is not negligible. This ion is high spin with a theoretical number of 5 unpaired electron. In a pure complex one should have an average of 3 unpaired electron per iron ion. Due to possible impurity a deviation can occur as in the case of 3,38 unpaired electrons. Noteworthy is the result of 0.94 unpaired electrons in $\mathrm{Ni}(\mathrm{II})$ cyclam. In a pure octahedral surrounding one would expect a high spin Ni(II) with two unpaired. The cavity inside the cyclam is too small for this high spin ion. Therefore a smaller low spin configuration is preferred. This has its effect on the geometry transforming from octahedral to square planar surrounding. This effect is described in literature.[9] The stability constant $\mathrm{K}$ equals 2.29 at room temperature. The equilibrium between square planar/ octahedral : 70\% / 30\%. This calculation leads to a theoretical result of 0.6 unpaired electron. In our case the equilibrium is displaced in favour of the octahedral form: $53 \% / 47 \%$.In this section clearly state results of the study or experiment.

When we take a closer view to the results obtained by analysing the solid complexes, The interpretation of results from analysis of the solid products by susceptometer measurements is less straight forward. First of all both the metal chlorides and the metal cyclam complexes are measured. When the results are plotted as $\chi_{\mathrm{mT}}$ as a funtion of $\mathrm{T}$, one can see that they generally obey the Curie Law as can be seen in figure 1 .

Fig.1 Susceptometer results for the metal cyclamcomplexes

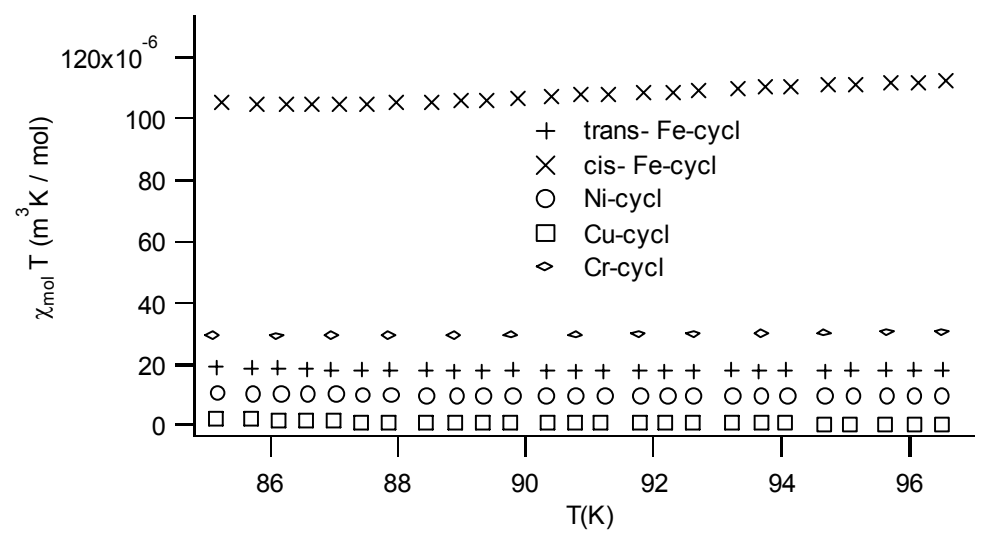

The calculated numbers of unpaired electrons are tabulated in table 1. The discrepancies between the theoretical and experimental values are much higher than in the case of the Evans'Method. Noteworthy is the fact that the equilibrium occurring with the Ni(II)cyclam towards square planar form is almost frozen in the octahedral crystalline form. Obviously, in the solid state, the complexes do no longer have the freedom to equilibrate between the octahedral and square planar environments and the apical occupancy is fixed with the chlorine anions. Again, large differences between the theoretical and experimentally values are seen for the Fe complexes. For the trans complex, the same explanation is valid as that used in the analysis of the Evans'method. In case of the $\mathrm{Fe}(\mathrm{III}) \mathrm{Cl}_{3}$ and the cis-Fe-cyclam the calculated number of unpaired electrons exceeds the maximum number of 5 for the individual $\mathrm{Fe}(\mathrm{III})$ species. There is also the deviation from the Curie Law. These observations might point to ferrimagnetic behaviour of these Fe(III)complexes in the solid state. The presence of ferrimagnetic fractions was also suggested after analysis of the temperature dependence for the cis-Fe-cyclam in more detail. Indeed, as figure 5 shows, the well known deviation from linearity allows us to prove the presence of ferrimagnetic phases in this compound. 


\section{Discussion}

As can be extracted from the two previous parts, it is necessary to compare the two methods and highlight the differences. In the case of the $\mathrm{Cr}(\mathrm{III})$-ion the results obtained from the dissolved species match better the expected values. With $\mathrm{Ni}$ (II) the opposite result is obtained. Here, one has to take into account equilibrium behaviour between the octahedral and square planar geometry, the reorganisation of the complex in aqueous solution which isn't possible in the solid state. The results of $\mathrm{Fe}(\mathrm{III}) \mathrm{Cl}_{3}$ and the cis-Fe(III) cyclam complex exhibit an overshoot in the solid complex. This can be explained by the presence of ferrimagnetic species as mentioned in the previous paragraph. This ferrimagnetic behaviour doesn't occur in solution because of the large influence of entropy at room temperature. The interpretation of the results obtained for the trans-Fe(III) cyclam complex is more difficult because of the presence of cis-isomers. The possibility to transform from trans to cis-isomer in the dissolved form is an acceptable theory. At last, the solid form of the $\mathrm{Cu}$ complexes leads to a smaller amount of unpaired electrons. But in the case of $\mathrm{CuCl}_{2}$, a smaller discrepancy is noted by the susceptometer measurement, while the NMR based Evans' method lies closer to the theoretically expected value in case of the cyclam complex.

\section{Conclusions}

Using two independent methods, it was possible to obtain detailed information about the changes in electron configuration occurring when cyclam complexes of transition metal ions are crystallized from aqueous solutions. Some of these changes could be attributed to effects due to the local geometry, others point to the existence of cooperative magnetic interactions which are typical for the solid state. Finally, in the case of the iron metal salts and their cyclam complexes large discrepancies occur.

\section{References}

[1] Martell AE, Hancock RD, Modern Inorganic Chemistry, ed JP Fackler jun. Plenum Press, New York, 1995.

[2] Dhont K, Herman G, Fabretti A, Lippens W, Goeminne A: Protonation and metal-ion complexation in aqueous solution by pyridine-containing hexaaza macrocycles, Journal of the Chemical Society, Dalton Transactions, 1996, (8) :1753-1760.

[3] Blain S, Appriou P, Chaumeil H, Handel H, Analytica Chimica Acta, 1990, 232 (2) 331336.

[4] Löliger J, Scheffold R, Journal of Chemical Education, 1972,49 (9), 646-647.

[5] Bosnich B, Tobe MI, Webb GA, Inorganic Chemistry, 1965, 4 (8), 1109-1112.

[6] Chen Y, Perkovic MW, Inorganica Chemica Acta, 2001,317 (1-2) 127-132.

[7] Guilard R, Siri O, Tabard A e.a., Journal of the Chemical society : Dalton Transactions, 1997, (19), 3459-3463

[8] Cotton, A.F. Progress in Inorganic Chemistry, Interscience Publishers : New York, 1964.

[9] Fabbrizzi, L.; Montagna, L. Journal of the Chemical society : Dalton Transactions, 1987, $11,2631-2634$. 